\title{
Dispepsia: caracterização e abordagem
}

\section{Dyspepsia: clinical aspects and current approach}

\author{
Fernando Marcuz Silva ${ }^{1}$
}

\begin{abstract}
Silva FM. Dispepsia: caracterização e abordagem. Rev Med (São Paulo). 2008 out.dez.;87(4):213-23.

RESUMO: A dispepsia é uma síndrome de alta prevalência, embora somente uma pequena parte dos pacientes que sofrem com ela procure atenção médica. Os conceitos e abordagens relacionadas à síndrome, apoiados nas sugestões do Consenso de Roma III, são apresentados neste artigo. Casos da síndrome na prática médica são propostos para auto-avaliação, e suas soluções e comentários são também apresentados.
\end{abstract}

DESCRITORES: Dispepsia. Úlcera gástrica. Refluxo gastroesofágico. Úlcera Péptica. Neoplasias gástricas.

\section{Caracterização}

A síndrome dispéptica, um problema atual comum e universal, é caracterizada por sintomas relacionados ao aparelho digestório alto. É manifestação de diferentes doenças, mas principalmente das doenças pépticas, ou seja, das doenças determinadas pela disfunção cloridropéptica: a doença de refluxo gastro-esofágico (DRGE), a úlcera péptica gastroduodenal e a dispepsia funcional ${ }^{7}$.

\section{Conceitos e sintomas}

Os sintomas que a caracterizam são sempre sintomas relacionados ao aparelho digestório alto: mais pertinentes como a dor epigástrica e o desconforto pós-prandial; mais sugestivos de doenças do esôfago: como a pirose retroesternal, a azia, a regurgitação, a disfagia e a odinofagia; inespecíficos como a eructação excessiva e a aerofagia; ou ainda os de base fisiopatológica mais ampla como as náuseas e os vômitos ${ }^{33}$.

O consenso de Roma $\mathrm{II}^{6}$, direcionado para as doenças funcionais do aparelho digestório sugere que, para o diagnóstico de dispepsia, sejam considerados como sintomas de dispepsia apenas a dor epigástrica: sensação subjetiva e desagradável que os pacientes sentem quando está havendo lesão tecidual, restrita a região do epigástrio, a pirose epigástrica: sensação desagradável de queimação limitada à região do epigástrio, a plenitude pósprandial: sensação desagradável que a comida permanece prolongadamente no estômago; e a saciedade precoce: sensação que o estômago fica cheio logo depois de iniciar a comer, desproporcional

\footnotetext{
${ }^{1}$ Médico Assistente do Serviço de Clínica Geral e Propedêutica, Divisão de Clínica Médica do Hospital das Clínicas da Faculdade de Medicina da Universidade de São Paulo - HC-FMUSP, Doutorando em Gastroenterologia pela FMUSP. Endereço para correspondência: Fernando Marcuz Silva. Hospital das Clínicas da FMUSP - ICHC - PAMB - 4ํ․ Andar, sala 6. Av. Dr. Enéas de Carvalho Aguiar, 155. Cerqueira César. São Paulo, SP. CEP: 05 403-900. E-mail: fmarcuz@ hcnet.usp.br
} 
ao volume ingerido, tanto que não se consegue terminar a refeição. Estes sintomas poderiam ter um significado fisiopatológico mais específico para dispepsia, enquanto os demais estariam definindo outras síndromes.

O consenso propõe ainda diferentes tipos de dispepsia: a dispepsia funcional, em que os sintomas não estão relacionados a doenças de base orgânica e os achados de endoscopia são normais ou menores (gastrite); a dispepsia orgânica, em que os sintomas dispépticos estão relacionados a uma doença orgânica, como a úlcera péptica; e a dispepsia não diagnosticada, quando os sintomas dispépticos ainda não foram investigados e para a qual o consenso propõe apenas algumas regras gerais de abordagem.

Propõe-se ainda que, quando os sintomas predominantes do paciente sejam pirose retrosternal, azia (sensação de regurgitação ácida ou azeda) ou regurgitação, anteriormente definindo a dispepsia tipo refluxo, ele seja diagnosticado como portador da DRGE e abordado como tal. É verdade que, para o diagnóstico desta doença, não há um exame padrão ouro, e cerca de metade dos casos são de DRGE não erosiva, que à endoscopia digestiva poderiam ser confundidos com casos de dispepsia funcional ${ }^{9}$.

Os sintomas de náuseas e vômitos são comuns, com um extenso diagnóstico diferencial e com fisiopatologia não necessariamente afeito a um sintoma dispéptico do tipo dismotilidade gástrica e, por isso mesmo, quando não associados uma doença orgânica definida, devem ser considerados num grupo fisiopatológico diferente da dispepsia: Distúrbios de Náusea e Vômitos Funcionais.

Apesar da dor abdominal na dispepsia ser uma dor do tipo visceral, portanto sem uma relação direta com o sitio anatômico afetado, o consenso também propõe que dores localizadas fora do epigástrio não sejam consideradas sintomas dispépticos. Localizadas no andar superior do abdômen, quando no hipocôndrio direito são mais sugestivas de doença biliar, e a investigação vai exigir ultra-sonografia. Quando no hipocôndrio esquerdo pode estar mais relacionada ao intestino.

Sintomas do aparelho digestório alto associados as do aparelho digestório baixo também não devem ser considerados como sintomas de dispepsia, porque a síndrome do intestino irritável se sobrepõe à dispepsia, com fisiopatologia semelhante, existindo mesmo quem considere que ela deva ser chamada de síndrome do aparelho digestório irritável ${ }^{27}$.

O uso de antiinflamatórios e alguns outros medicamentos podem determinar lesões gastrintestinais, mais freqüentemente úlceras e erosões, principalmente de características agudas.
Podem ser acompanhadas de sangramento digestivo e caracterizam uma doença orgânica bem definida: lesões gastrintestinais por medicamentos e assim não devem ser abordadas, em princípio, como quadros de dispepsia².

\section{A dispepsia não diagnosticada}

O Consenso de Roma III está focado na dispepsia funcional. Assim, para critérios diagnósticos, propõe as restrições sintomáticas acima e define ainda uma duração de sintomas que devem ser estar presente por pelo menos 3 meses, com pelo menos 6 meses do início dos sintomas. Para a dispepsia não diagnosticada, não se tem definida uma duração mínima, embora alguns autores considerem pelo menos 4 semanas nos últimos 3 meses. $O$ consenso Roma III é menos específico e propõe como orientações gerais:

- Certificar-se de que os sintomas são restritos ao trato digestivo alto;

- Identificar os sinais ou sintomas de alarme: perda de peso inexplicada, vômitos recorrentes, disfagia progressiva, sangramento gastrointestinal, anemia, visceromegalia, etc., que não são comuns na prática diária e que podem ter valor preditivo positivo para doenças orgânicas. Quando presentes ditam a indicação de endoscopia digestiva de início;

- Certificar-se do possível uso de antiinflamatórios não esteroidais;

- Caracterizar como uma Doença de Refluxo Gastro-Esofágico a presença de sintomas típicos de refluxo.

De acordo com as condições do local, indicar a endoscopia digestiva de início para pacientes idosos.

\section{O Helicobacter pylori na dispepsia}

O Helicobacter Pylori (H. pylorı) é uma bactéria espiralada, flagelada, gram-negativa, microaerófila, que consegue infectar o estômago e sobreviver no ambiente ácido graças a sua capacidade de produção de urease e de alcalinização do seu microambiente. Tem alta prevalência no mundo todo, com taxas mais altas nos países desenvolvidos, quando comparado com os em desenvolvimento ${ }^{3}$. No nosso meio esta taxa é de $65 \%$ da população ${ }^{34}$. A contaminação ocorre principalmente na infância e dificilmente ocorre eliminação espontânea da bactéria, o que determina uma infecção crônica em todos os infectados. A maioria das pessoas é assintomática, mas uma pequena parte da população pode desenvolver úlcera péptica, linfoma tipo MALT e câncer gástrico ${ }^{22}$.

A erradicação da bactéria previne a recidiva 
da úlcera, pode curar o linfoma gástrico MALT de pequeno grau e prevenir o câncer gástrico. Apenas uma parte dos pacientes que apresentam dispepsia funcional e são portadores da bactéria apresentam remissão dos sintomas quando tratados da infecção. Assim, é possível que os sintomas dispépticos em pacientes com gastrite pelo $H$. pylori não se correlacionem com a infecção ${ }^{14}$.

\section{O câncer gástrico na dispepsia}

O câncer não é uma doença péptica, mas por se situar no estômago pode determinar sintomas dispépticos. Via de regra, o câncer gástrico, quando apresenta sintomas dispépticos, se apresenta numa forma avançada e dificilmente nesta fase pode apresentar possibilidade de cura $^{28}$. Porém, dada a gravidade da doença, a demora em seu diagnóstico, quando de sintomas dispépticos, é um fator de desconforto para o médico, para o paciente e seus familiares. Assim é um motivo de discussão especial a abordagem de pacientes dispépticos idosos e ou com antecedentes familiares da doença $a^{13,15}$.

No entanto, mesmo nos países com alta prevalência de câncer gástrico (Japão, Coréia, Portugal, Albânia, etc.), dificilmente a porcentagem de pacientes com dispepsia não diagnosticada é maior que 5\%, quando submetida ao exame endoscópico ${ }^{32}$.

\section{Epidemiologia}

A prevalência da síndrome é muito alta na população em geral, em torno de $40 \%$, porém somente um quarto dos pacientes procura cuidados médicos por sua causa. No Brasil, um estudo de base populacional ${ }^{4}$ revelou uma prevalência de $44 \%$ utilizando os critérios de Roma $I^{5}$ e $19,5 \%$ utilizando os critérios de Roma II ${ }^{30}$.

Nos Estados Unidos, um estudo mostrou que a mais freqüente causa de dispepsia é a dispepsia funcional, e que a prevalência de dispepsia é de 31,9\% e, ao se excluir os casos de refluxo (Critérios de Roma II e Roma III), diminuiu para $15,8 \%{ }^{23}$.

Para os serviços de atenção primária, sintomas de dispepsia são muito mais freqüentes, embora grande parte destes pacientes apresente sintomas dispépticos associados a doenças não pépticas como Síndrome do Intestino Irritável, Pancreatopatias, Síndrome de Má-absorção, Doença Inflamatória Intestinal, Endocrinopatias, Distúrbios Hidroeletrolíticos, Ansiedade, Depressão e outras (Quadro 1).A síndrome dispéptica, especialmente por causa da dispepsia funcional, tem baixa morbidade, porém apresenta grande impacto na qualidade de vida e nos custos de sua investigação, tratamento $e$ absenteísmo do trabalho ${ }^{16}$.

QUADRO 1. Causas de dispepsia

\begin{tabular}{|l|}
\hline Digestivas pépticas \\
\hline Dispepsia funcional \\
Doença de refluxo gastro-esofágico \\
Úlcera Péptica \\
\hline Digestivas não pépticas \\
Gastropatias específicas \\
(Tuberculose, Citomegalovirose, Sarcoidose, Doença de Crohn) \\
Neoplasias \\
(Gástrica, Pancreática, de Cólon) \\
Síndromes de má absorção \\
(Doença celíaca) \\
Colelitíase \\
\hline Não digestivas \\
Doenças metabólicas \\
(Diabete melito, Doenças da tiróide, Hiperparatiroidismo, Distúrbios eletrolíticos) \\
Doença coronariana \\
Colagenoses \\
Medicamentos \\
(Antinflamatórios não esteroidais, Antibióticos, Xantinas, Alendronato) \\
Doenças psiquiátricas \\
(Ansiedade, Depressão, Pânico, Distúrbios alimentares) \\
\hline
\end{tabular}


A dispepsia funcional é a causa mais freqüente da dispepsia não investigada (Quadro 2). A prevalência da úlcera péptica vem diminuindo (exceto a forma hemorrágica em idosos ${ }^{8}$ ), enquanto a DRGE vem aumentando, provavelmente pela queda da prevalência da infecção pelo H. pylorie pelo aumento da obesidade. Há uma maior preocupação hoje em dia com as complicações da DRGE, já que parece estar havendo um aumento da incidência do adenocarcinoma da junção gastro-esofágica nos países ocidentais ${ }^{18}$ e mesmo no Japão ${ }^{10}$.

QUADRO 2. Achados endoscópicos em pacientes dispépticos

\begin{tabular}{|c|c|c|c|c|c|}
\hline Fonte & $\begin{array}{c}\text { Pacientes } \\
n\end{array}$ & Refluxo \% & $\begin{array}{c}\text { Úlcera } \\
\%\end{array}$ & $\begin{array}{c}\text { Câncer } \\
\%\end{array}$ & $\begin{array}{c}\text { Funcional } \\
\%\end{array}$ \\
\hline Ayoola et al. & 10.112 & 11 & 17 & 1,5 & 70 \\
\hline Hungin et al. & 6.744 & 17 & 15 & 0,6 & 67 \\
\hline Hallisey et al. & 2.659 & 19 & 16 & 2,7 & 62 \\
\hline Fedail et al. & 2.500 & 8 & 18 & 2,2 & 71 \\
\hline Mansi et al. & 2.086 & 5 & 13 & 1,5 & 81 \\
\hline Holdstock et al. & 1.805 & 9 & 14 & 2,0 & 75 \\
\hline Fjosne et al. & 1.275 & 10 & 23 & 4,0 & 62 \\
\hline Capurso et al. & 1.1 .53 & 3 & 8 & 0,6 & 89 \\
\hline Stanghellini et al. & 1.057 & 9 & 21 & 0,6 & 69 \\
\hline Davenport et al. & 1.041 & ND & 24 & 2,6 & ND \\
\hline Nyren et al. & 972 & 3 & 23 & 0,3 & 74 \\
\hline Gear et al. & 968 & 5 & 17 & 2,3 & 76 \\
\hline Joahnnessen et al. & 930 & 15 & 17 & 1,0 & 67 \\
\hline Bytzer et al. & 878 & 2 & 15 & 1,4 & 82 \\
\hline Talley et al. & 820 & 14 & 23 & 3,4 & 60 \\
\hline Williams et al. & 686 & 14 & 28 & 2,5 & 56 \\
\hline Saunders et al. & 559 & 31 & 23 & 0,2 & 46 \\
\hline Intervalo (\%) & & $2-31$ & $13-28$ & $0,2-4,0$ & $35-89$ \\
\hline Média (\%) & & 12 & 17 & 1,6 & 70 \\
\hline
\end{tabular}

Modificado de Arents et al. ${ }^{1}$

\section{Abordagem}

A dispepsia pode ser abordada de formas diferentes. A rigor, características da população, prevalência da infecção pelo $H$. pylori, prevalência do câncer gástrico, custos e disponibilidade da endoscopia digestiva alta devem ser adequadamente estudados para cada região. Também os métodos diagnósticos não invasivos para a bactéria e outros fatores (inclusive também a vontade do paciente) devem ser levados em conta na adoção de uma estratégia específica.
$\mathrm{Na}$ prática, três estratégias são mais comumente utilizadas: Endoscopia de início, identificação da infecção pelo $H$. pylori e sua erradicação (estratégia "teste e trate") e tratamento empírico inicial.

\section{Endoscopia inicial}

A endoscopia de início tem sido proposta como abordagem que faz o diagnóstico da dispepsia precocemente. Tranqüiliza o paciente ${ }^{19}$ e identifica tanto os casos de dispepsia funcional (a maioria dos 
casos), que são benignos e não vão exigir muitos recursos no seu acompanhamento, bem como os casos de neoplasia gástrica. Porém, a endoscopia digestiva é um exame que tem restrições de oferta e, em alguns países, tem alto custo, de maneira que economizar sua indicação muitas vezes é uma necessidade real ${ }^{17}$.

\section{Teste e trate}

A estratégia do "teste e trate", que consiste em erradicar $\circ H$. pylori em pacientes dispépticos jovens e sem sinais de alarme, apresenta uma relação custo benefício melhor que a endoscopia inicial e pode economizar até $30 \%$ de exames ${ }^{11}$. Porém é necessária a disponibilidade dos testes não invasivos para o $H$. pylori. A prevalência da infecção também não pode ser alta ou muito baixa, porque ou se erradicaria a bactéria em quase todos os dispépticos ou em muito poucos e mesmo em pacientes ulcerosos, a erradicação da bactéria pode não acabar com os sintomas ${ }^{26}$.

\section{Tratamento empírico}

O tratamento empírico inicial (prova terapêutica) é uma estratégia também direcionada para pacientes dispépticos jovens e sem sinais de alarme, que propõe um tratamento empírico, normalmente utilizando supressores ácidos, quem com resposta adequada do pacientem é mantida por algum tempo, visando a resolução dos sintomas ${ }^{20}$. Para os pacientes que não respondem ao tratamento, a endoscopia está indicada e, para os pacientes cujos sintomas recidivam, também. É verdade que cerca de metade dos casos de dispepsia funcional são recidivantes, mas o princípio da abordagem permite o diagnóstico das úlceras pépticas e das doenças de refluxo, que respondem ao tratamento de supressão ácida, mas sempre recidivam.

Também é importante salientar que, para esta estratégia (também a do teste e trate), o reconhecimento dos sinais de alarme (sinais ou sintomas que denotam gravidade em doença orgânica) indica endoscopia inicial ${ }^{12}$ (vide os principais sinais de alarme no Quadro 3).

De maneira geral, quase todos os trabalhos apontam a idade como um sinal de alarme, variando a faixa etária proposta. O ideal é que cada localidade faça estudos para determiná-la. No Brasil normalmente é adotada a faixa etária dos Estados Unidos: 55 anos (Quadro 4). Em princípio, para localidades com alta prevalência de câncer gástrico, recomendam-se faixas etárias mais baixas ${ }^{31}$.

\section{QUADRO 3. Sinais e sintomas de alarme em dispepsia}

\begin{tabular}{|l|}
\hline Emagrecimento inexplicado \\
Anemia \\
Sangramento digestivo \\
Disfagia progressiva \\
Vômitos persistentes \\
Cirurgia gástrica prévia \\
Visceromegalia \\
Icterícia \\
\hline Tumoração ou adenopatia abdominal \\
Sintomas sistêmicos \\
Idade (1) \\
Uso de antiinflamatórios (2) \\
(1) verificar a faixa etária; (2) sem gravidade, não considerar
\end{tabular}
alarme.
QUADRO 4. Abordagem da dispepsia pelo tratamento empírico

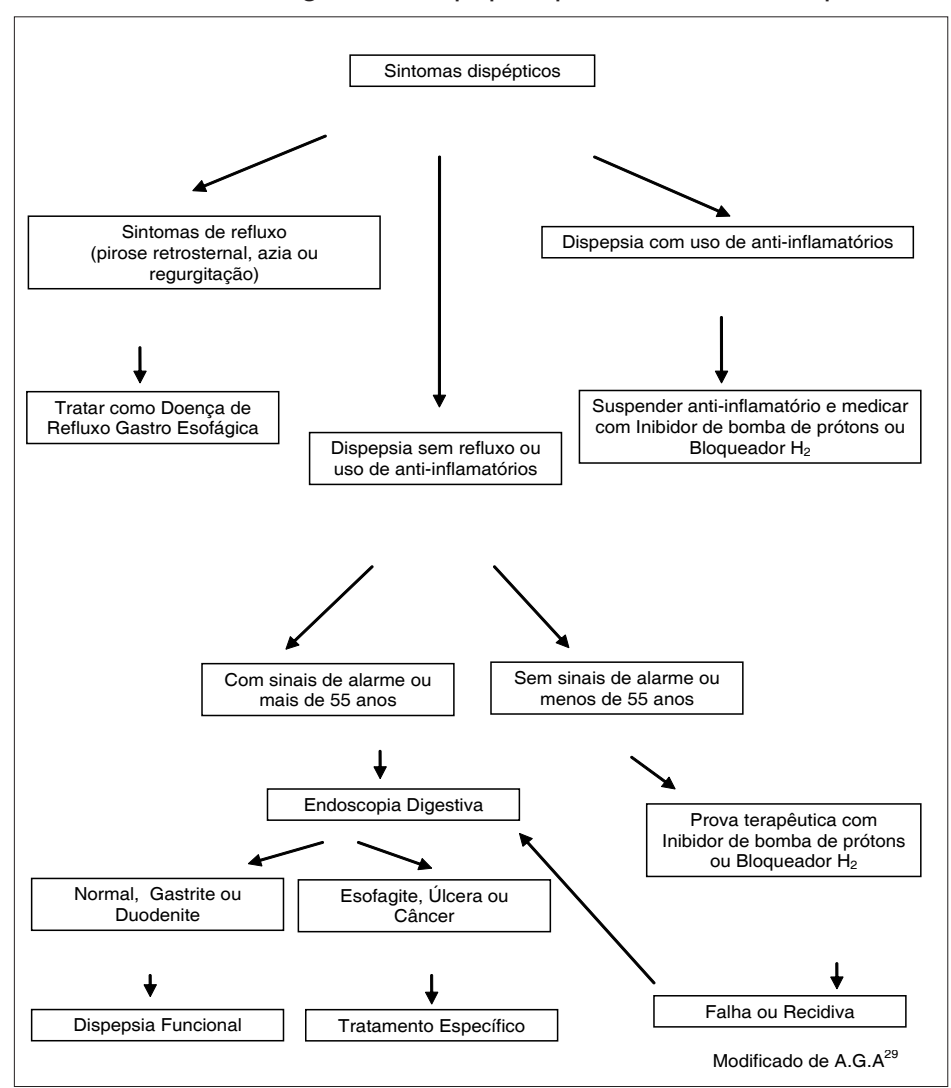


Também o uso de antiinflamatório pode ser considerado um sinal de alarme, porque este quadro normalmente é pouco sintomático e pode estar associado à maior morbidade e mortalidade, em especial em pacientes idosos. Porém, desde que não haja gravidade hemodinâmica, a suspensão do medicamento e o uso de inibidor de bomba de prótons podem proporcionar rápida cicatrização das lesões ${ }^{25}$, sem necessidade de endoscopia.

\section{A úlcera péptica}

A úlcera péptica tem sido o apanágio da dis- pepsia, tanto que a dispepsia funcional também é conhecida como dispepsia não ulcerosa. Tem como causa principalmente a infecção pelo H. pylori, o uso de antiinflamatórios e, muito raramente, situações de hipergastrinemia, como a síndrome de ZollingerEllison ${ }^{24}$. Diversas patologias determinam úlceras no aparelho digestório alto, porém há causas não pépticas, como o câncer gástrico, o linfoma, a doença de Crohn, a tuberculose, o citomegalovírus e outros.

O seu tratamento consiste na supressão do uso de anti-inflamatórios e na erradicação do $H$. pylori (Quadro 5). A supressão ácida determina apenas a cicatrização temporária das úlceras (Quadro 6).

QUADRO 5. Esquemas para tratamento do Helicobacter pylori

\begin{tabular}{|c|c|c|c|}
\hline \multicolumn{4}{|c|}{ primeiro tratamento } \\
\hline $\mathrm{IBP}^{*}$ & $2 X$ & 7 dias & \\
\hline Amoxi 1000mg & $2 X$ & 7 dias & $85 \%(109)$ \\
\hline Claritro 500mg & $2 x$ & 7 dias & \\
\hline \multicolumn{4}{|c|}{ re-tratamento } \\
\hline IBP & $1 \mathrm{X}$ & 7 Dias & \\
\hline Furazolidona 200mg & $3 X$ & 7 dias & $75 \%^{(112)}$ \\
\hline Tetraciclina 500mg & $3 X$ & 7 dias & \\
\hline
\end{tabular}

* (PyloriPac, Heliclar, HelicoPac, Erradic = produtos com o tratamento completo e embalagem calendário oferecidos no mercado)

QUADRO 6. Supressores ácidos

\begin{tabular}{|c|c|c|c|}
\hline \multicolumn{4}{|c|}{ Inibidores de bomba de prótons } \\
\hline Substância & Dose usual & $1 / 2$ dose & dose dupla \\
\hline Omeprazol & $20 \mathrm{mg}$ & $10 \mathrm{mg}$ & $40 \mathrm{mg}$ \\
\hline Lansoprazol & $30 \mathrm{mg}$ & $15 \mathrm{mg}$ & \\
\hline Pantoprazol & $40 \mathrm{mg}$ & $20 \mathrm{mg}$ & \\
\hline Rabeprazol & $20 \mathrm{mg}$ & $10 \mathrm{mg}$ & \\
\hline Esomeprazol & $40 \mathrm{mg}$ & $20 \mathrm{mg}$ & \\
\hline \multicolumn{4}{|l|}{ Bloqueadores H2 } \\
\hline Cimetidina & $800 \mathrm{mg}$ & $400 \mathrm{mg}$ & \\
\hline Ranitidina & $300 \mathrm{mg}$ & $150 \mathrm{mg}$ & \\
\hline Nizatidina & $300 \mathrm{mg}$ & $150 \mathrm{mg}$ & \\
\hline Famotidina & $40 \mathrm{mg}$ & $20 \mathrm{mg}$ & \\
\hline \multicolumn{4}{|l|}{ Antiácidos } \\
\hline Hidóxido de alumínio & $200 \mathrm{mg}(4 \mathrm{x} \text { ao dia })^{*}$ & & $200 \mathrm{mg} 7 \mathrm{x}$ ao dia** \\
\hline Hidróxido de magnésio & $200 \mathrm{mg}(4 \times \text { ao dia })^{*}$ & & $200 \mathrm{mg} 7 \times$ ao dia** \\
\hline Hidróxido de alumínio & $200 m g+200 m g(4 x \text { ao dia })^{*}$ & & $200 \mathrm{mg}+200 \mathrm{mg}$ \\
\hline e magnésio & & & $7 \times$ dia $^{* *}$ \\
\hline
\end{tabular}

*Ministrar duas horas após as refeições e ao deitar

** Ministrar uma hora antes e duas horas após as refeições e ao deitar 


\section{A doença de refluxo gastro-esofágico}

De fisiopatologia ainda não bem determinada, parece decorrer do desbalanço entre os mecanismos de defesa contra o refluxo gástrico e, principalmente, a acidez do refluído gástrico. O esfíncter inferior do estômago é a mais importante barreira contra o refluxo, e a hipotonia episódica deste esfíncter é a principal causa da doença. É possível ocorrer doença extra-digestiva (asma, faringite e laringite de refluxo, sem esofagite concomitante). O tratamento é baseado principalmente no uso do inibidor de bomba de prótons e nas modificações do estilo de vida: elevação da cabeceira da cama, redução do peso corpóreo, abstenção do álcool, tabaco e medicamentos agressivos (alendronato, sildenafila, anti-inflamatórios), dieta sem irritantes e fracionada ${ }^{24}$.

\section{A dispepsia funcional}

A mais freqüente causa de dispepsia ainda não tem sua fisiopatologia bem definida, embora seja uma doença benigna e a investigação endoscópica mostre mucosa gastroduodenal normal ou com lesões mínimas (gastrite). Talvez seja multi-causal, e a agressão cloridro-péptica, a hipersensibilidade visceral, a dismotilidade gastroduodenal e distúrbios psicológicos e do sistema nervoso central estejam envolvidos ${ }^{24}$.

Seu tratamento pode ser difícil e não há medicamentos de grande eficácia a serem usados. Uma proposta para a abordagem medicamentosa é apresentada no Quadro 7.

QUADRO 7. Tratamento da dispepsia funcional

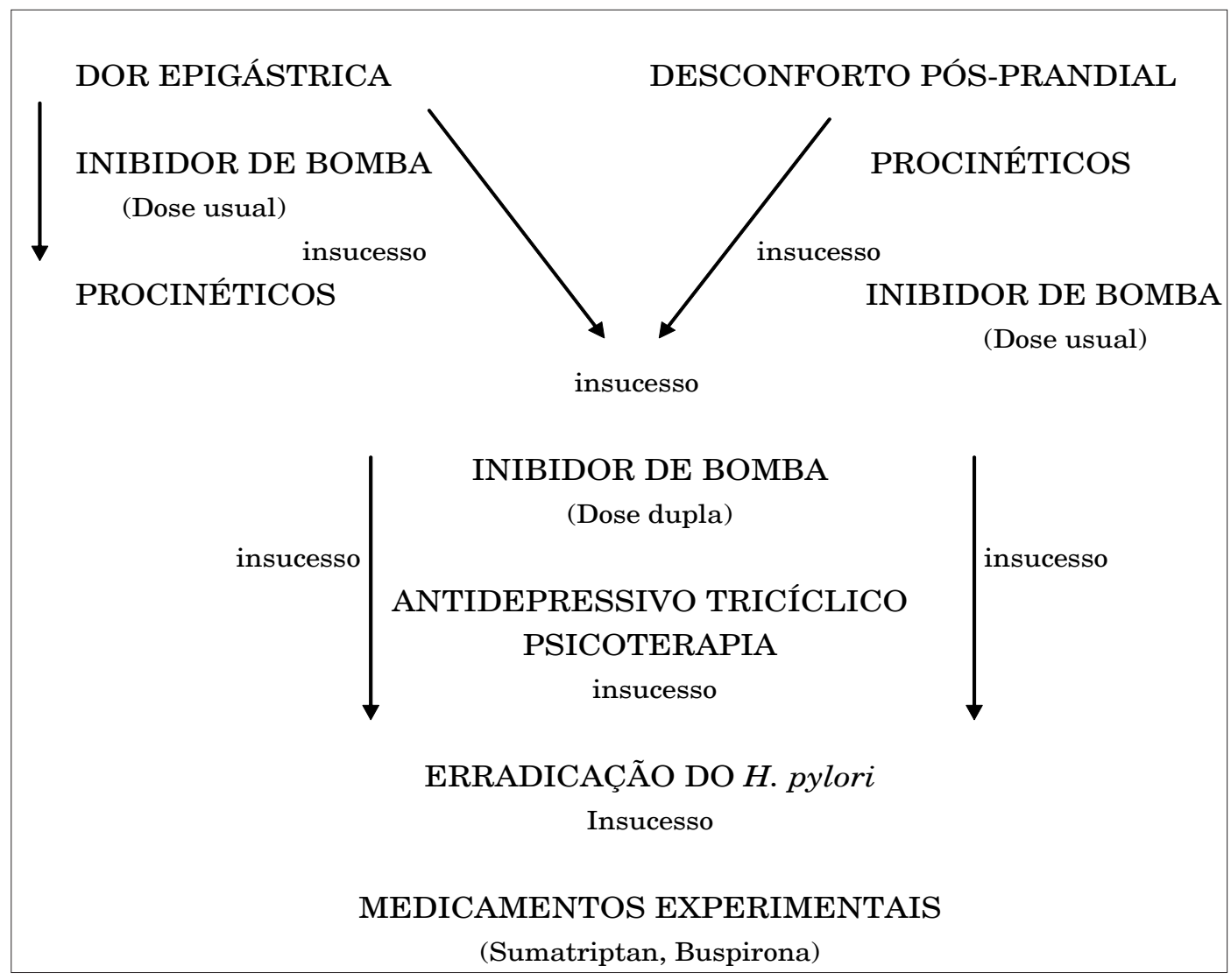

Tratamento comportamental visando a utilização de uma dieta saudável, sem irritantes, evitar o uso de medicamentos agressivos para a mucosa gástrica e minimizar as situações de ansiedade ou depressão podem ser úteis, embora não hajam estudos adequados verificando a eficácia destas medidas.

É sempre muito importante compreender a limitação do uso de medicamentos, dar suporte psicológico ao paciente, conscientizando-o de que a doença é benigna e aguardar o surgimento de medicamentos mais eficazes ${ }^{21}$. 


\section{Casos problemas - resolução}

1. Paciente feminina de 16 anos refere desconforto epigástrico há 3 meses, que piora com a ingestão de alimentos gordurosos. Tem náuseas freqüentes pela manhã. Nega vômitos. Nega emagrecimento, uso de anti-inflamatórios, álcool e tabaco. Nega passado mórbido. É muito emotiva e reconhece que os sintomas são mais intensos e freqüentes quando fica ansiosa. Caso de dispepsia sem sinais de alarme em paciente jovem. A proposta é realizar um tratamento empírico com supressores ácidos por 4 a 8 semanas. Se a paciente não responder ao tratamento ou em caso de recidiva após tratamento, indicar endoscopia. Verificar a necessidade de tratamento da ansiedade.

2. Paciente masculino de 52 anos tem dor epigástrica há 5 anos, em crises de meses de duração, que melhora com a alimentação. Refere "clocking" freqüente. É pedreiro e tabagista de 20 cigarros por dia. Nega perda de peso. Há 1 semana evacuou durante 3 dias fezes escuras e de mau cheiro. Refere melhora da dor com a ingestão de leite ou de bicarbonato.

Caso de dispepsia com sinal de alarme: sangramento digestivo. Indicar endoscopia o mais breve possível. Medicar se possível com inibidor de bomba de prótons. Verificar o uso de anti-inflamatório, porque o paciente tem uma profissão sujeita a lesões osteo-musculares, situação de uso freqüente destes medicamentos.

O paciente tem os sintomas recidivantes e clocking, o que pode ser mais sugestivo de úlcera péptica. $\mathrm{O}$ paciente tem dor típica: melhora com alcalinos, porém usa bicarbonato, medicamento que é absorvido e pode determinar alterações eletrolíticas sistêmicas. É tabagista, hábito que aumenta a freqüência de recidiva ulcerosa e aumenta o tempo de cicatrização da úlcera. Esta característica pode ser minimizada se o paciente for portador da infecção pelo $H$. pylori e erradicar a infecção.

3. Paciente masculino de 65 anos, natural de Portugal, no Brasil há 10 anos. Ex-fumante, abstêmio há 5 anos, sempre gostou de alimentos defumados, com baixa ingesta de verduras. Há 3 meses apresenta epigastralgia leve, sem passado dispéptico prévio, que piora com a ingestão de alimentos, sem náuseas ou vômitos, porém com anorexia. Perdeu $2 \mathrm{~kg}$ de peso no período.

Dispepsia com sinal de alarme: perda de peso, em paciente idoso, procedente de um país com alta prevalência de câncer gástrico, com dispepsia de início recente. Também tem hábitos de risco para câncer gástrico. Solicitar endoscopia o mais procecemente possível. Medicar se possível com inibidor de bomba de prótons.

4. Paciente feminina de 45 anos refere há um ano queimação retroesternal, que piora após as refeições e é mais intensa à noite, dificultando o seu sono. Tem azia constante e episódios repetidos de regurgitação.
Apresenta intolerância aos gordurosos e ao álcool. É obesa e não consegue emagrecer. Fuma e não quer interromper o hábito porque tem medo de engordar. Já fez diversos tratamentos com antiácido ou cimetidina, com melhora transitória (enquanto está usando o medicamento).

Caso sugestivo de DRGE sem sinais de alarme. Medicar com inibidor de bomba de prótons por seis meses, orientar medidas comportamentais: elevação da cabeceira da cama, evitar deitar logo após as refeições, dieta fracionada, seca, em especial sem bebidas gasosas, diminuição da ingesta de café e gordurosos, estimular a abstenção de álcool e tabaco, orientar e apoiar as medidas para perda de peso. A paciente tem melhora transitória com uso de supressão ácida de menor potência. É possível que eventualmente tenha necessidade de usar dose dobrada de inibidor de bomba de prótons e que a suspensão do uso deste tipo de medicamentos se siga de recidiva precoce dos sintomas. Nesta situação poderá ser candidata ao uso crônico de inibidor de bomba de prótons. Resposta inadequada a dose dobrada do medicamento por mais de um mês de tratamento, é indicação de endoscopia e reavaliação do diagnóstico. Em pacientes mulheres de mais idade verificar o uso de alendronato.

5. Paciente feminina de 21 anos refere epigastralgia de forte intensidade, que piora com as refeições e com qualquer tipo de alimento. Está fazendo uso de omeprazol por orientação médica há uma semana, sem melhora. Há 6 meses teve episódio semelhante, quando fez endoscopia digestiva que mostrou uma gastrite enantemática, $H$. pylori $\oplus$. Melhorou com uso de bromopride. Quer repetir a endoscopia, porque como acha a dor atual muito mais intensa, acredita que a gastrite se transformou em úlcera.

Caso de dispepsia funcional. O tratamento é sintomático e a supressão ácida potente não necessariamente melhora os sintomas. Às vezes o uso de procinéticos pode ser mais eficaz. Verificar a necessidade de se tratar ansiedade ou depressão associada. A erradicação do $H$. pylori não está indicada, porque até o momento não há evidências que a erradicação da bactéria melhore os sintomas. Não há necessidade de se repetir a endoscopia, porque a intensidade dos sintomas não se correlaciona com a gravidade da gastrite e a não ser em caso de mudança dos sintomas o quadro dispéptico recidivante está associado ao mesmo achado endoscópico. Estes casos são os mais difíceis de se tratar na prática médica: demandam apoio médico constante, cuidado em não supervalorizar medicamentos e exames laboratoriais e aconselhamento continuado para uma vida saudável e de menor preocupação com os sintomas dispépticos, porque a entidade é benigna.

6. Paciente masculino de 51 anos tem epigastralgia há 10 anos. Já fez inúmeras endoscopias, que sempre mostram úlcera duodenal (às vezes, mais de uma). Obtém melhora transitória com uso de ranitidina, porém quando suspende a medicação volta a ter 
dor. Há 2 meses foi orientado a fazer um tratamento para erradicação do $H$. pylori. Usou corretamente a medicação, porém apresentou recidiva dolorosa 60 dias depois do tratamento.

Caso de úlcera duodenal, provavelmente $H$. pylori dependente. É necessário verificar o uso de antiinflamatórios, uma outra causa de recidiva ulcerosa. Porém a cura da úlcera associada à infecção pelo $H$. pylori só ocorre com a erradicação da bactéria. Como a reinfecção em adultos é muito rara, a não melhora dos sintomas após o tratamento da infecção ou está associada ao uso de anti-inflamatórios ou à resistência da bactéria ao esquema terapêutico utilizado. É importante no retratamento da bactéria utilizar antibióticos diferentes dos usados previamente e com esquemas testados para a nossa população. Hoje em dia, no nosso meio, o esquema inicial preferencial utiliza inibidor de bomba de prótons, amoxicilina e claritromicina 2 vezes ao dia por 7 dias. Há diversas apresentações comerciais com embalagem-calendário utilizando este esquema. No retratamento, uma opção barata e eficaz é a associação de omeprazol, furazolidona e tetraciclina 2 vezes ao dia por 7 dias. Com este esquema é importante a orientação dos pacientes para os efeitos adversos da furazolidona que é um medicamento IMAO-símile. O controle de tratamento da infecção, quando necessário (úlceras complicadas) pode ser feito com o exame: Teste Respiratório com Uréia Marcada com Carbono-13, se a endoscopia não é necessária e deve preferencialmente ser realizado de 60 a 90 dias após o uso do tratamento.

7. Paciente masculino de 41 anos apresenta há 1 ano crises de epigastralgia, acompanhada de náuseas e vômitos, que associa ao tratamento de crises de podagra, que apresenta com freqüência. Para tratamento da artrite se utiliza de diclofenaco, que toma 3 a 4 vezes por dia. No último episódio, quando apresentou melena, foi submetido à endoscopia, que mostrou úlcera gástrica ativa, com sinais de sangramento recente.

Caso de úlcera associada ao uso de anti-inflamatório. O paciente deve evitar usar este tipo de medicamento. Às vezes é preferível o uso de corticosteróides (que também apresentam riscos) ao uso de antiinflamatórios não esteroidais. Os anti-inflamatórios COX2 seletivos tem menor potencial ulcerogênico, porém não devem ser usados prolongadamente, em especial em idosos e pacientes com aterosclerose, por causa do risco potencial de desencadeamento de eventos cardio-vasculares. O uso concomitante de supressores ácidos não protege totalmente do surgimento de úlcera ou gastrite erosiva. A recidiva ulcerosa em pacientes tratados com anti-inflamatórios em uso de bloqueador $\mathrm{H} 2$ é de $50 \%$ e em uso de inibidor de bomba de prótons, $25 \%$. Não há efeito adicional de proteção com doses maiores que $20 \mathrm{mg}$ de omeprazol (e equivalente a de outros inibidores de bomba de prótons). No tratamento deste paciente (com sangramento ativo) é mais adequado, se possível, medicá-lo com dose dobrada de inibidor de bomba de prótons, que nesta dose consegue cicatrizar $87 \%$ das úlceras em 15 dias. Também é adequado realizar precocemente a erradicação do $H$. pylori, porque o tratamento da bactéria em úlcera hemorrágica previne ressangramentos.

8. Paciente feminina de 70 anos, portadora de HAS e DM foi tratada de infarto agudo do miocárdio. Na alta foi prescrito AAS 100mg por dia e como a paciente sabe ser portadora de úlcera duodenal, foi prescrito concomitantemente ranitidina $300 \mathrm{mg} / \mathrm{dia}$.

Caso de úlcera péptica em paciente idosa que tem indicação de profilaxia com AAS em baixa dosagem. É sabido que doses de $50 \mathrm{mg}$ de AAS já podem inibir a ciclo-oxigenase e aumentar e o risco de doença gastrointestinal associada a este medicamento. Se absolutamente necessário é possível tentar a proteção gástrica com o uso concomitante de inibidor de bomba de prótons na dose usual. Hoje em dia, para pacientes que apresentam doença gástrica associada ao uso de anti-inflamatório em baixa dosagem, pode-se tentar o uso do clopidogrel ou da ticlopidina, porém estes medicamentos também podem determinar complicações gastrointestinais.

9. Um paciente de 45 anos tem história de azia e pirose retrosternal, associadas à episódios de chiado no peito e tosse seca. Vem usando omeprazol 20mg/ dia com controle parcial dos sintomas. Submetido à endoscopia digestiva teve diagnóstico de esofagite erosiva e esôfago de Barret.

Caso de DRGE com manifestação extra-digestiva: asma e faringite por refluxo. Além disso, na investigação do paciente foi detectado o esôfago de Barret, uma complicação da doença de refluxo: uma transformação metaplásica da mucosa esofágica, associada ao refluxo gastro-esofágico, que predispõe ao adenocarcinoma do esôfago. Para doenças extra-digestivas do refluxo se propõe a prescrição de inibidor de bomba de prótons em dose dobrada por pelo menos 6 meses, associado às medidas comportamentais anti-refluxo. No caso de esôfago de Barret o uso do inibidor de bomba de prótons deve ser indefinido e deve ser realizado periodicamente exame endoscópico para o rastreamento do adenocarcinoma de esôfago.

10. Um paciente com episódio de melena refere sintomas de dor epigástrica há 6 meses, que controlava com uso intermitente de omeprazol, sem orientação médica. A endoscopia mostrou úlceras duodenais em beijo e uma úlcera na segunda porção do duodeno. A pesquisa do H. pylori foi negativa e gastrinemia de $900 \mathrm{pg} / \mathrm{ml}$. O paciente tem antecedente de nefrolitíase.

Caso de úlcera duodenal associada à hipergastrinemia. O uso de inibidor de bomba de prótons pode determinar hipergastrinemia acentuada. Embora gastrinemia acima de $1.000 \mathrm{pg} / \mathrm{mL}$ seja indicativa de gastrinoma, a determinação deste exame deve ser realizada com a suspensão do medicamento por pelo menos 7 dias antes de sua coleta, o que pode repre- 
sentar uma dificuldade técnica importante, já que o paciente apresenta úlcera ativa e com necessidade de supressão ácida potente. Além disso, a presença da infecção pelo $H$. pylori também pode determinar alteração da gastrinemia e o uso de inibidor de bomba de prótons também podem mascarar a presença da infecção. No entanto a presença de úlceras múltiplas em especial mais distalmente ao estômago também podem sugerir hiperacidez gástrica. $O$ paciente tem história de nefrolitíase e no caso de gastrinoma associado à síndrome NEM (neoplasia endócrina múltipla), a hipercalcemia associada ao adenoma de paratireóide surge normalmente antes do aparecimento do gastrinoma. Este tipo de gastrinoma tem carater familiar e frequentemente apresenta múltiplos pequenos tumores na parede duodenal. No entanto, a Síndrome de Zollinger-Ellison (gastrinomas associados à úlcera péptica e hipersecreção gástrica) é uma síndrome muito rara. Neste caso seria adequado realizar nova dosagem da gastrina em situação ideal, pesquisar e erradicar o $\mathrm{H}$. pylori, se presente, medicar o paciente por um período mínimo de 4 semanas com inibidor de bomba de prótons em dose dobrada e se a situação clínica se mantiver, encaminhar o paciente para a especialidade para a pesquisa de gastrinoma. Gastrimenias entre 100 e 500 pg/mL são mais sugestivas de hiposecreção gástrica, freqüente em gastrites atróficas.

Silva FM. Dyspepsia: clinical aspects and current approach. Rev Med (São Paulo). 2008 out.dez.;87(4):213-23.

\begin{abstract}
Dyspepsia is a syndrome with a high prevalence in general population, but only few patients seek medical care by his symptoms. The concepts and the approaches related to syndrome, supported by Roma III Consensus, are presented in this article. Clinical cases in medical practice are proposed for self evaluation and the solutions with proper commentaries are presented also.
\end{abstract}

KEY WORDS: Dyspepsia. Stomach ulcer. Gastroesophageal reflux. Peptic ulcer. Stomach neoplasms.

\section{REFERÊNCIAS}

1. Arents NL, Thijs JC, Kleibeuker JH. A rational approach to uninvestigated dyspepsia in primary care: review of the literature. Postgrad Med J. 2002;78(926):707-16.

2. Bjarnason I, Scarpignato C, Takeuchi K, Rainsford KD. Determinants of the short-term gastric damage caused by NSAIDs in man. Aliment Pharmacol Ther. 2007;1:26(1):95-106.

3. Coelho LG, Leon-Barua R, Quigley EM. Latin-American Consensus Conference on Helicobacter pylori infection. Latin-American National Gastroenterological Societies affiliated with the Inter-American Association of Gastroenterology (AIGE). Am J Gastroenterol. 2000;95(10):2688-91.

4. de Oliveira SS, da Silva dos Santos I, da Silva JF, Machado EC. Prevalence of dyspepsia and associated sociodemographic factors. Rev Saude Publica. 2006;40(3):420-7.

5. Drossman DA. The functional gastrintestinal disorders: diagnosis, phatophysiology and treatment. A multinational consensus. Mclean, VA: Degnon Associates; 1994.

6. Drossman DA. The functional gastrointestinal disorders and the Rome III process. Gastroenterology. 2006;130(5):1377-90.
7. Ford AC, Moayyedi P. Current guidelines for dyspepsia management. Dig Dis. 2008;26(3):225-30.

8. Kang JY, Elders A, Majeed A, Maxwell JD, Bardhan $\mathrm{KD}$. Recent trends in hospital admissions and mortality rates for peptic ulcer in Scotland 1982-2002. Aliment Pharmacol Ther. 2006;1:24(1):65-79.

9. Keohane J, Quigley EM. Functional dyspepsia and nonerosive reflux disease: clinical interactions and their implications. Med Gen Med. 2007;9(3):31.

10. Kusano C, Gotoda T, Khor CJ, Katai H, Kato H, Taniguchi $\mathrm{H}$, et al. Changing trends in the proportion of adenocarcinoma of the esophagogastric junction in a large tertiary referral center in Japan. J Gastroenterol Hepatol. 2008;23(11):1662-5.

11. Lassen AT, Hallas J, Schaffalitzky de Muckadell OB. Helicobacter pylori test and eradicate versus prompt endoscopy for management of dyspeptic patients: 6.7 year follow up of a randomised trial. Gut. 2004;53(12):1758-63.

12. Lieberman D, Fennerty MB, Morris CD, Holub J, Eisen G, Sonnenberg A. Endoscopic evaluation of patients with dyspepsia: results from the national endoscopic data repository. Gastroenterology. 2004;127(4):1067-75. 
13. Liou JM, Lin JT, Wang HP, Huang SP, Lee YC, Shun $\mathrm{CT}$, et al. The optimal age threshold for screening upper endoscopy for uninvestigated dyspepsia in Taiwan, an area with a higher prevalence of gastric cancer in young adults. Gastrointest Endosc. 2005;61(7):819-25.

14. Mazzoleni LE, Sander GB, Ott EA, Barros SG, Francesconi CF, Polanczyk CA, et al. Clinical outcomes of eradication of Helicobacter pylori in nonulcer dyspepsia in a population with a high prevalence of infection: results of a 12-month randomized, double blind, placebo-controlled study. Dig Dis Sci. 2006;51(1):89-98.

15. Mimica M. Choice of age cut-off for endoscopy in dyspepsia in developing countries according to incidence of gastric cancer. Coll Antropol. 2005;29(2):599-602.

16. Mones J, Adan A, Segu JL, Lopez JS, Artes M, Guerrero T. Quality of life in functional dyspepsia. Dig Dis Sci. 2002;47(1):20-6.

17. Ofman JJ, Rabeneck L. The effectiveness of endoscopy in the management of dyspepsia: a qualitative systematic review. Am J Med. 1999;106(3):335-46.

18. Pera M. Trends in incidence and prevalence of specialized intestinal metaplasia, Barrett's esophagus, and adenocarcinoma of the gastroesophageal junction. World J Surg. 2003;27(9):999-1008; discussion 6-8.

19. Quadri A, Vakil N. Health-related anxiety and the effect of open-access endoscopy in US patients with dyspepsia. Aliment Pharmacol Ther. 2003;17(6):835-40.

20. Rabeneck L, Souchek J, Wristers K, Menke T, Ambriz E, Huang I, et al. A double blind, randomized, placebo-controlled trial of proton pump inhibitor therapy in patients with uninvestigated dyspepsia. Am J Gastroenterol. 2002;97(12):3045-51.

21. Saad RJ, Chey WD. Review article: current and emerging therapies for functional dyspepsia. Aliment Pharmacol Ther. 2006;24(3):475-92.

22. Sanders MK, Peura DA. Helicobacter pylori-associated diseases. Curr Gastroenterol Rep. 2002;4(6):448-54.

23. Shaib Y, El-Serag HB. The prevalence and risk factors of functional dyspepsia in a multiethnic population in the United States. Am J Gastroenterol. 2004;99(11):2210-6.

24. Silva FM. Dispepsia. In: Santos IS, Silva LL, Bensenor IM, editores. Clínica médica - diagnóstico e tratamento. São Paulo: Sarvier; 2008. p. 857-61.

25. Singh G, Triadafilopoulos G. Appropriate choice of proton pump inhibitor therapy in the prevention and management of NSAID-related gastrointestinal damage. Int J Clin Pract. 2005;59(10):1210-7.

26. Spiegel BM, Vakil NB, Ofman JJ. Dyspepsia management in primary care: a decision analysis of competing strategies. Gastroenterology. 2002;122(5):1270-85.

27. Spiller R, Aziz Q, Creed F, Emmanuel A, Houghton $L$, Hungin $P$, et al. Guidelines on the irritable bowel syndrome: mechanisms and practical management. Gut. 2007;56(12):1770-98.

28. Sundar N, Muraleedharan V, Pandit J, Green JT, Crimmins R, Swift GL. Does endoscopy diagnose early gastrointestinal cancer in patients with uncomplicated dyspepsia? Postgrad Med J. 2006;82(963):52-4.

29. Talley NJ. American Gastroenterological Association medical position statement: evaluation of dyspepsia. Gastroenterology. 2005;129(5):1753-5.

30. Talley NJ, Stanghellini V, Heading RC, Koch KL, Malagelada JR, Tytgat GN. Functional gastroduodenal disorders. Gut. 1999;45(Suppl 2):II37-42.

31. Uehara G, Nago A, Espinoza R, Vargas G, Astete M, Moran L, et al. Optimal age for gastric cancer screening in patients with dyspepsia without alarm symptoms. Rev Gastroenterol (Peru). 2007;27(4):339-48.

32. Uemura N, Okamoto S, Yamamoto S. H. pylori infection and the development of gastric cancer. Keio $\mathrm{J}$ Med. 2002;51(Suppl 2):63-8.

33. Vakil N, van Zanten SV, Chang L, Toth G, Sherman $\mathrm{J}$, Fraser $\mathrm{M}$, et al. Comprehension and awareness of symptoms in women with dyspepsia. Aliment Pharmacol Ther. 2005;22(11-12):1147-55.

34. Zaterka S, Eisig JN, Chinzon D, Rothstein W. Factors related to Helicobacter pylori prevalence in an adult population in Brazil. Helicobacter. 2007;12(1):82-8. 